\title{
The relationship between self-esteem and self-forgiveness: Understanding the mediating role of positive and negative self-compassion
}

Ruchi Pandey ${ }^{1}$, Gyanesh Kumar Tiwari ${ }^{1}$, Rakesh Pandey ${ }^{2}$, Satchit Prasun Mandal ${ }^{3}$, Sujata Mudgal ${ }^{4}$, Priyanka Parihar ${ }^{1}$, Pramod Kumar Rai ${ }^{1}$, Ari Sudan Tiwari ${ }^{5}$, and Meenakshi Shukla ${ }^{6}$

${ }^{1}$ Department of Psychology, School of Humanities \& Social Sciences, Doctor Harisingh Gour Vishwavidyalaya, Sagar, 470003, Madhya Pradesh, India

${ }^{2}$ Department of Psychology, Faculty of Social Sciences, Banaras Hindu University, 221005, Varanasi, Uttar Pradesh, India

${ }^{3} 4$ Air Force Selection Board, Defence Research and Development Organization (DRDO), Varanasi, Uttar Pradesh, India

${ }^{4}$ School of Humanities and Social Sciences, G. D. Goenka University, Sohna, Gurgaon, 122001, Haryana, India

${ }^{5}$ Defence Institute of Psychological Research, Defence Research and Development Organization (DRDO), 110054, New Delhi, India

${ }^{6}$ Department of Psychology, Magadh University, Bodh Gaya, 824234, Bihar, India

May 18, 2020

\begin{abstract}
Self-esteem and self-compassion represent well-known positive self-resources with significant implications for life outcomes of people belonging to individualistic and collectivistic cultures, respectively. Both the constructs have been suggested to shape the nature and extent of self-forgiveness through dissimilar mechanisms. The study examined the mediating role of positive and negative self-compassion between the relationship between self-esteem and self-forgiveness. Employing a convenient sampling, 144 male $(\mathrm{M}=22.10, \mathrm{SD}=1.66)$ and 124 female participants $(\mathrm{M}=21.98, \mathrm{SD}=1.90)$ were chosen for a correlational research design. The findings showed that Self-Esteem and Positive Self-Compassion had significant positive correlations with Self-Forgiveness. Conversely, Negative Self-Compassion exhibited negative correlations with these measures. Self-esteem and Positive Self-Compassion accounted for significant positive variance in Realization \& Reparation and Overall Self-Forgiveness and significant negative variability in Attribution. Negative Self-Compassion accounted for positive significant variance in Guilt and negative significant variability in Realization \& Reparation and Attribution. Both Positive and Negative Self-Compassion significantly mediated the relationships among Self-Esteem and Realization \& Reparation, Guilt and Attribution. It evinced coexistence and working of self-esteem and self-compassion in a collectivistic culture with more pronounced effects of the later. Re-conceptualization of self-compassion is recommended.
\end{abstract}

Keywords

self-esteem; positive and negative self-compassion; self-forgiveness; mediation analysis; path analysis. 


\section{Introduction}

Forgiveness represents a human virtue that reflects lack of resentment and involves benevolence, compassion and love towards a transgressor (Thompson et al., 2005; Worthington, 2001). The researchers have reported forgiveness to be facilitative for the development and restoration of self and interpersonal relationships (McCullough, 2008), mental health and well-being (Griffin et al., 2020; J. R. Webb \& Toussaint, 2020; E. L. Worthington \& Scherer, 2004) and physical health (Lawler et al., 2003; Toussaint et al., 2020). It has also been found to be an effective intervention method for interpersonal and emotional problems and promotes well-being (Baskin \& Enright, 2004). The impacts and benefits of forgiveness are extended from intrapersonal, interpersonal and collective to organizational levels (Aquino et al., 2006; Noor et al., 2008). Many antecedents and correlates have been identified to unearth the nature and dynamics of forgiveness. Many intrapersonal and interpersonal factors such as perceived severity of transgression (Fincham et al., 2005); absence of apology (McCullough et al., 1998), the nature of relationship (McNulty, 2011), emotions (McCullough et al., 1998), cognitions (McCullough et al., 2007) and personality attributes (Berry et al., 2005) have been found to be closely linked with forgiveness.

There are various forms of forgiveness that involve oneself, others, deity, society, and situations (Toussaint \& Webb, 2005). Although a good number of studies have been conducted on interpersonal forgiveness, there has been a dearth of scientific studies on self-forgiveness. With its effectiveness in clinical interventions and the development of psychometric measures, self-forgiveness has recently attracted the attention of the researchers (Scherer et al., 2011; Wohl et al., 2008). Self-forgiveness refers to the desire to give up anger and grudges directed towards self as a result of perceived wrongdoings of one's own on the one hand and to cultivate compassion, self-kindness and care toward oneself on the other (Enright, 1996). Thus, self-forgiveness initiates a set of motivational changes that inbreed preventive and promotive strengths enabling a person to enhance positive cognition, behaviour and affect for self and affected others (Hall \& Fincham, 2005).

Based on the interpersonal model of forgiveness, Hall and Fincham (2005) have proposed the first model that suggested self-forgiveness to originate from shame and guilt that are caused by attributions and the severity of the transgressions. They further argued that guilt and conciliatory behaviours toward a victim and perceived forgiveness may facilitate or inhibit self-forgiveness. Empirical validation of the model showed that attributions, empathy, and shame were unrelated to self-forgiveness, and only transgression severity, guilt, conciliatory behaviours, and perceived forgiveness influenced the development of self-forgiveness beyond the effect of time (Hall \& Fincham, 2008).

Rangganadhan and Todorov (2010) reported Hall and Fincham's model of self-forgiveness(Hall \& Fincham, 2005) inadequate and put forth their own model in which guilt positively affects self-forgiveness via prompting both other-oriented empathy and conciliatory behaviour, while shame negatively affects selfforgiveness, both individually and by increasing personal distress empathy, or the experience of self-oriented distress at the recognition of another's discomfort. McConnell et al. (2012) tested the original model proposed by Hall and Fincham for the third time and observed that the alternative model, which included severity of the offence, guilt, conciliatory behaviour, and perceived forgiveness from the victim, best accounted for the variance in self-forgiveness. McGaffin et al. (2013) integrated the findings of all the three previous models of self-forgiveness and suggested that guilt and shame lead to two distinct consequences. For example, guilt promotes other-oriented empathy, conciliatory behaviours and acceptance while shame may lead to acceptance and personal distress empathy. It provided for acceptance as a predictor of self-forgiveness and distinguished between other-oriented empathy and personal distress empathy.

All these models of self-forgiveness have much in common and they all provide for the importance of transgression severity, shame, guilt, conciliatory behaviours, and perceived forgiveness from the victims. Most recently, a model of self-forgiveness has been presented by Mudgal and Tiwari (2017) who employed exploratory research design, a variant of mixed methods design, on Indian population and came up with three components of self-forgiveness namely, realization \& reparation, guilt and attribution. The uniqueness 
of this model lies in the empirical validation of these components of self-forgiveness which suggested it to be significant predictors of general health and quality of life of the adults (Mudgal \& Tiwari, 2017b). Self-forgiveness has been reported to be positively related to the positive health outcomes and decreased depression and anxiety (Maltby et al., 2001), and self-esteem, well-being, close-relationships, self-activity and social engagement in female victims (Coates, 1996), less suicidal behaviour(Hirsch et al., 2011), physical and mental health (Jon R. Webb et al., 2013), greater correctional involvement (Biron, 2007), lower levels of neuroticism and greater agreeableness (Biron, 2007; Butzen, 2009).

\section{Self-Esteem and Self-Forgiveness}

It has been found that the individuals who experience an attack on their self-esteem are less able to forgive. It is the self-esteem that ascertains the level of threat due to any transgressions, it may be argued to be important for the study of forgiveness (Strelan \& Zdaniuk, 2015). Researchers have also suggested that there is a need to test the process driving the relationship between self-esteem and self-forgiveness (Strelan \& Zdaniuk, 2015).

The lower level of self-esteem encourages people to enter self-protection and develop avoidance that may affect self-forgiveness negatively. It has been reported that excess self-protection caused by poor self-esteem may decrease the accessibility of psychological resources needed for forgiveness to come out (Strelan \& Zdaniuk, 2015). The severity of transgression has been reported to impede forgiveness in many studies (Fincham et al., 2005; Griffin et al., 2020) that necessitates the assessment of the roles of the positive self resources in regulating forgiveness (Strelan \& Zdaniuk, 2015).

A perusal of previous studies evinced that little is known about the extent to which an individual's self-esteem affects forgiveness (Strelan \& Zdaniuk, 2015). Some studies have been conducted employing trait self-esteem with mixed findings. A meta-analytic review showed that there was a very weak relationship between trait self-esteem and situation-specific forgiveness (Fehr et al., 2010). It may be argued that the transgression situation in which the individuals commit wrongdoing may also affect their self-esteem and may later impact their forgiveness. Some researchers have suggested that people use self-esteem as a resource (Steele et al., 1993) that acts as a standard or expectancy that is guided by their values and situational cues. It has been argued that although people try to maintain specific positive self-images, the primary motivation behind this image is to maintain global self-integrity, a general perception of their goodness, virtue, and efficacy. Thus, having high self-esteem facilitates individuals to affirm their resources and lesser use of defensive mechanisms or distort reality. Through their self-worth, people can have positive attitudes towards self and others. Moreover, individuals with high self-esteem are also more likely to acknowledge their own personal responsibility in the face of adversity, pain and wrongdoings. It has been argued that selfesteem originates from social comparison and denotes one's ranking. It reflects one's sense of self-worth and evaluations(Harter, 1999). It has also been argued that having high self-esteem is not always beneficial for individuals. Some ill-consequences of having higher self-esteem have also been identified by some researchers. For example, having higher self-esteem may involve puff the self up in putting others down, lead to a grandiose view of oneself and self-esteem may result from narcissism(Neff, 2011). Thus, high self-esteem originating from narcissism may lead to negative outcomes (anger, retaliation) while healthy self-esteem may culminate in positive ones (MacKinnon, 2015).

\section{Self-Compassion and Self-Forgiveness}

Borrowed from Buddhist philosophy, self-compassion entered recently in the literature of Psychology. Self-compassion refers to a set of positive self-attitudes and behaviours that enhances the positive relationship with the self and self-worth that originates in the face of adversity, pain and failures. Self-compassion has been suggested to comprise of three interdependent and interrelated bipolar dimensions: Self-kindness vs. self-judgement, common humanity vs. Isolation and mindfulness vs. Over-identification. The first dimension is characterized by self-care and positive attitudes in adversity while the second facilitates the understanding 
that pain or failure is common experiences to all. The third aspect represents to become aware of one's negative experiences and refrain from denying it (Neff, 2003a, 2003b). In essence, self-compassion denotes self-attitude, feelings and behaviours in the face of adversity, pain or failure of life. It is one of the significant self-resources that has attracted the attention of researchers in recent past and has been reported to be closely linked with positive health outcomes, academic success, well-being and performance (Rai \& Tiwari, 2019; Verma \& Tiwari, 2017).

It has been reported that self-compassion and self-forgiveness are closely linked but their nature of association has not been understood properly (Conway-Williams, 2015). Self-forgiveness and selfcompassion are similar in their definitions, components, processes and contributions to various life outcomes (Conway-Williams, 2015). An analysis of the definitions of these constructs showed that fostering compassion, generosity, humanity and love toward oneself involve in the constructs of self-forgiveness and self-compassion (Enright, 1996; Worthington et al., 2005). These two also have similarities in regulating a broad range of psychological functioning and are assumed to carry adaptive role in self-regulation, pro-social and positive virtues (Berry et al., 2005), health functioning (Christensen et al., 2011) and well-being (Pandey et al., 2019, 2020).

These arguments evinced that self-compassion and self-forgiveness are linked closely but the empirical validation of their association is lacking (Conway-Williams, 2015). The search on PsycINFO in 2014 identified only three studies that reported their relationships with limited aspects of human behaviours (Conway-Williams, 2015). (Neff and Pommier (2013)examined the relationship between self-compassion and forgiveness of others in undergraduate students, older adults, and active mediators and reported it to be correlated with forgiveness of others in all three samples. There is a clear theoretical rationale for a direct relationship between self-compassion and self-forgiveness (Conway-Williams, 2015). Exploring the nature of the relationship between self-compassion and self-forgiveness may be important for the integration of these two areas of research as well as for furthering understanding of each of these constructs independently. It may be argued that self-compassion may impact one's inclination to forgive oneself. Moreover, self-compassion is more frequent and relevant variable and comparatively easy to experience than self-forgiveness. In addition, cognitive, emotional, and experiential responses towards committing an offence may be linked with selfcompassion that, in turn, may affect the likelihood of self-forgiveness. Self-compassion may lead one to experience less rumination, shame, and experiential avoidance that may facilitate self-forgiveness.

Self-compassion and self-forgiveness occur at the time when an individual is facing adversity, pain and guilt. Both the processes are closely associated with self-knowledge and self-attitude. Both the processes have positive impacts on the individuals. Thus, they must be linked with each other. In spite of the pivotal role of these two psychological constructs, there is a dearth of studies relating these two constructs. Self-compassion has been suggested to be positively related to well-being and negatively linked with psychopathological symptoms (MacBeth \& Gumley, 2012; Muris et al., 2018). Very recently, some researchers have shown their disagreements with the empirical validity of components of self-compassion model proposed by Neff (2003a) and questioned the suitability and usefulness of incorporating self-judgement, isolation and overidentification to her model (Muris et al., 2016). Muris and Petrocchi (2017) argued that self-kindness, common humanity and mindfulness (positive aspects of self-compassion) exhibit negative associations with mental health problems whereas self-judgement, isolation and over-identification (negative dimensions of self-compassion) show with psychopathological symptoms. Similar observations were also made by some other researchers who reported comparable findings regarding positive and negative self-compassion (López et al., 2015; Pfattheicher et al., 2017).

Neff and associated researchers of self-compassion conducted the further statistical analysis and came to their previous conclusions that bifactor model reflects the most suitable model of self-compassion (Cleare et al., 2018; Neff et al., 2017). These efforts did not satisfy the opponents who questioned the inclusion of positive and negative dimensions in the proposed model of self-compassion. The opponents argued that selfjudgment, isolation and over-identification represent negative attributes and carry a close connection with self-criticism (Zuroff et al., 1990), social withdrawal and loneliness (Rubin \& Coplan, 2004), and self-focused 
rumination (Lyubomirsky \& Nolen-Hoeksema, 1995). Following this line of arguments, Muris et al. (2018) have recently testified the face and empirical validity of positive and negative dimensions of self-compassion proposed by (Neff, 2003b) and argued positive self-compassion to have a close association with adaptive coping and healthy functioning and negative self-compassion to be linked with maladaptive behaviours (Muris et al., 2018). Similar to the findings of Muris et al. (2018), other researchers have also observed a positive correlation between positive self-compassion and social support seeking, and reassuring thoughts and negative correlations with negative self-compassion (Allen \& Leary, 2010). Besides, negative self-compassion also showed its close association with passive reacting, avoidance and emotion suppression (Allen \& Leary, 2010). Similarly, positive self-compassion showed its positive association with adaptive coping strategies such as active coping, planning and positive reframing and negative relationships with maladaptive strategies such as behavioural disengagement and self-blame (Sirois et al., 2015).

Examining the association between self-compassion and self-forgiveness within more diverse populations has also been suggested (Conway-Williams, 2015). It is also needed to investigate the role of self-compassion in the self-forgiveness process (Wenzel et al., 2012). Majority of the study has employed three components model of self-compassion while ignoring the positive and negative dimensions which have very recently attracted the researchers. The findings suggest that conceptualization of self-compassion in terms of positive and negative dimensions are more efficacious to underscore the role of self-compassion in structuring the various life outcomes (Muris et al., 2018; Pandey et al., 2019). In the backdrop of these arguments, the present study aimed to explicate the mediating role of positive and negative dimensions of self-compassion between self-esteem and self-forgiveness with its three components, namely realization \& reparation, guilt and attribution proposed by Mudgal and Tiwari (2017).

\section{Objectives}

(1) To understand the nature and magnitude of correlations among Self-Esteem, Positive and Negative Self-Compassion and various components of Self-Forgiveness,

(2) To estimate the variance accounted for by Self-Esteem, and Positive and Negative Self-Compassion in the scores of various components of Self-Forgiveness, and

(3) To explore the mediating role of Positive and Negative Self-Compassion between the relationship of Self-Esteem and the three components of Self-Forgiveness (Realization \& Reparation, Guilt and Attribution).

\section{Hypotheses} formulated:

On the basis of the understanding of the available literature, the following hypotheses have been

(1) There will be positive correlations among Self-Esteem, Positive Self-Compassion, Realization \& Reparation, Attribution and Overall Self-Forgiveness while Negative correlation will be observed among the predictors and Guilt of the participants.

(2) There will be negative correlations among Negative Self-Compassion, Realization \& Reparation, Attribution and Overall Self-Forgiveness whereas a positive correlation will be recorded between Negative Self-Compassion and Guilt of the participants.

(3) The Self-Esteem and Positive Self-Compassion will account for positive variability in Realization \& Reparation, Attribution and Overall Self-Forgiveness while they will negatively contribute to the Guilt of the participants. On the hand, the Negative Self-Compassion will account for negative variability for Realization \& Reparation, Attribution and Overall Self-Forgiveness and strengthening variance for Guilt of the participants. 
(4) Positive and Negative Self-Compassion will mediate differently the relationship between Self-Esteem and Realization \& Reparation, Guilt and Attribution.

\section{Methods and Procedure}

\section{Participants}

The present study employed a convenient sampling method to choose the participants of the study. Initially, 351 participants pursuing their graduation and post-graduation programmes at schools of Arts, Commerce, Science and Law at Doctor Harisingh Gour Vishwavidyalaya (University), Sagar, Madhya Pradesh, India gave their consent with to participate. Out of these, only 300 participants with the age ranging from 18 years to 30 years $(\mathrm{M}=20.04, \mathrm{SD}=1.77)$ ascertained their final participation. The scores of these participants were screened for outliers through SPSS V25 as per the procedure outlined by Donald (2016). After the screening of the outliers, only data of 144 males (Age range $=18$ years to 25 years, $\mathrm{M}=22.10$, $\mathrm{SD}=1.66)$ and 124 females (Age range $=19$ years to 30 years, $\mathrm{M}=21.98, \mathrm{SD}=1.90$ ) were left for final analyses.

\section{Psychometric Tools}

The following psychometric tools were employed to collect the data of the study:

\section{Self-Esteem Scale}

Rosenberg Self-Esteem Scale is a unidimensional scale with 10-items that measures global selfworth by measuring both positive and negative feelings about the self (Rosenberg, 1965). The items seek an answer on a 4-point Likert scale format ranging from strongly agree to strongly disagree. It has been reported that people high in self-esteem show high attractiveness, better relationships and make better impressions on others. High self-esteem has also been shown to have a strong relation with happiness and lowered depression (Baumeister et al., 2003; Pandey et al., 2020).

\section{Self-Compassion Scale}

Self-compassion of the participants was measured with the help of the Self-Compassion Scale (Neff, 2003a). The items of the self-compassion measure carry the thoughts, emotions and behaviours representing the various components of self-compassion. The scale consists of 26 items that comprises Self-Kindness (items5, 12, 19, 23, 26), Self-Judgment (items-1, 8, 11, 16, 21), Common Humanity (items-3, 7, 10, 15), Isolation (items-4, 13, 18, 25), Mindfulness (items-9, 14, 17, 22) and Over-Identification (items-2, 6, 20, 24) with a five-point scale from almost never to almost always. The internal reliability of the measure has been reported to be satisfactory in many studies carried out across many populations (Allen et al., 2012; Neff \& Pommier, 2013; Werner et al., 2012). The translated versions of this measure have also observed a six-factor structure of the scale across cultures (Arimitsu, 2014; Azizi et al., 2013; Castilho et al., 2015; Garcia-Campayo et al., 2014).

\section{Self-Forgiveness Scale}

Standardized on Indian population with exploratory mixed methods design, the Self-Forgiveness Scale developed by Mudgal and Tiwari (2017) was used to assess the self-forgiveness of the participants. The self-forgiveness measure comprises of 30 items with a seven-point scale (1-very strongly disagree, 2-strongly disagree, 3 -somewhat disagree, 4- neutral, 5 -somewhat agree, 6 - strongly agree, 7 -very strongly agree). The scale represents three dimensions of Self -Forgiveness: Realization \& Reparation (19 items), Guilt (6 items) and Attribution ( 5 items). The reliability coefficients were estimated by computing Cronbach's Alpha for the three subscales and the overall scale of self-forgiveness were $0.90,0.70,0.62$ and 0.77 , respectively. Likewise, 
the validity was estimated by computing correlation between the scores of the overall self-forgiveness scale and overall quality of life (World Health Organization, 1996) that came to be 0.261 .

\section{Procedure}

The proposal of the study was submitted to the ethics committee of [BLINDED] India of approval. After the receipt of ethical approval, the scales were procured and the participants were consulted. Written consent was collected from each participant. Then, they were debriefed about the objectives of the study. The data was collected in the small groups of 5 to 10 participants at a time for accuracy and ease. Accordingly, the scales were supplied to each participant and asked to read the printed instructions on each scale. The scoring was completed as per the guidelines provided depicted in the manuals of the scales. No compensation was given to the participants for their participation in the study.

\section{Data Analysis Plan}

The collected data were arranged according to the need of the statistical analyses and the same were treated with the help of SPSS v25. The data analyses were carried out in two phases: preliminary analysis, and mediation \& path analyses. In the first phase, Coefficients of Correlation among self-esteem, self-compassion and self-forgiveness were carried out. Besides, hierarchical analyses assuming Self-Esteem and Self-Compassion (positive and negative self-compassion) as predictor variables and Self-Forgiveness with its three components (Realization \& Reparation, Guilt and Attribution) as the criterion variables were also computed. The mediation analysis and path analysis were carried out in the second phase using SPSS V25.

\section{Results}

The results have been presented in two sub-sections: preliminary analyses, and mediation and path analyses.

\section{Preliminary Analyses}

At the stage of preliminary analyses, zero-order correlations were conducted to understand the nature and extent of association among self-esteem, components of self-forgiveness (realization \& reparation, guilt \&attribution) as well as positive and negative self-compassion (Table 1).

Table 1. Correlation matrix among self-esteem, components of self-forgiveness and positive/negative selfcompassion

\begin{tabular}{lllll}
\hline & Self-esteem & Positive self-compassion & Negative self-compassion & Realization \& Reparation \\
Self-esteem & 1 & $.523^{* *}$ & $.269^{* *}$ & $.289^{* *}$ \\
Positive self-compassion & $.523^{* *}$ & 1 & $-.140^{*}$ & $.446^{* *}$ \\
Negative self-compassion & $.269^{* *}$ & $-.140^{*}$ & 1 & $-.180^{* *}$ \\
Realization \& Reparation & $.289^{* *}$ & $.446^{* *}$ & $-.180^{* *}$ & 1 \\
Guilt & .002 & $-.151^{*}$ & $.227^{* *}$ & $-.394^{* *}$ \\
Attribution & $-.148^{*}$ & $.150^{*}$ & $-.310^{* *}$ & $.218^{* *}$ \\
Overall Self-forgiveness & $.280^{* *}$ & $.442^{* *}$ & $-.165^{* *}$ & $.955^{* *}$ \\
\hline
\end{tabular}

The analyses revealed significant inter-correlations among Self-esteem and "Realization \& Reparation dimension", attribution as well as the total score of self-forgiveness. However, guilt did not yield significant correlation with Self-esteem. Similarly, positive and negative self-compassion differentially correlated with 
the dimensions of self-forgiveness. Positive self-compassion correlated positively and significantly with all the three dimensions of Self-forgiveness except Guilt whereas Negative Self-compassion followed an exactly opposite pattern of correlation. Correlation matrix also revealed that self-esteem correlated positively with both positive and negative self-compassion (see Table 1).

\section{Mediation and Path Analyses}

To investigate the mediating effect of positive and negative self-compassion between the relationship of self-esteem and self-forgiveness, a path model was constructed taking the positive and negative selfcompassion as mediators (Figure 1).

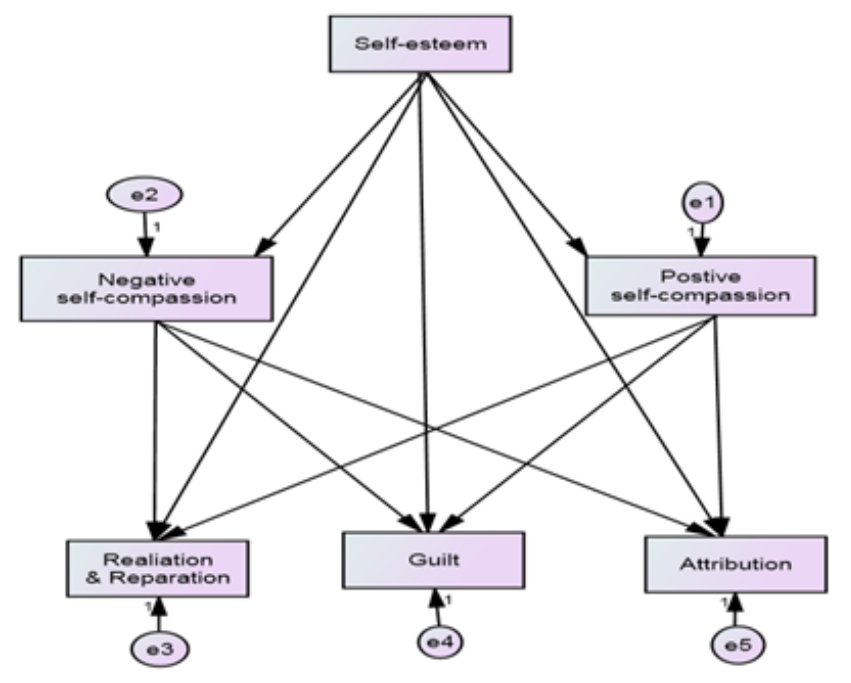

Figure 1: Figure 1 (Base Model): A path model taking positive and negative self-compassion as mediators in the relationship between self-esteem and self-forgiveness depicting (realization \& reparation, guilt \& attribution).

However, the base model (figure 1) was partially supported with standardized coefficients. The Chi-square $\mathrm{X}^{2}(\mathrm{df}=04, \mathrm{~N}=268)=82.5, p<.001$ reflected that the model was not a good fit to the data. The fit indices used in the study consist of commonly reported Comparative Fit Index (CFI) and Root Mean Square Error of Approximation (RMSEA). The CFI index compares the base model with the independence model: the values greater than .95 indicates a good-fitting model (Hu \& Bentler, 1999). The RMSEA assesses the comparability of the base model with an ideal (saturated) model. The more it approximates zero, the better the model would be. However, the values below .08 suggest a good fit. The hypothesized model (figure 1) displayed a poor CFI of .737 and RMSEA of .271. Another indication of the goodness of fit lies in the standardized residuals, which expected to be less than .080 in order to have a good-fit model. Present base model (figure 1) yielded an unacceptable value of standardized residuals (.113) which was beyond the acceptable limit. Likewise, remaining fit indices such as goodness of fit index (GFI, measures the effectiveness of the model in 
approximating the observed covariance matrix, cut off value >.95), created by Jöreskog and Sörbom (1998), reflected a satisfactory GFI value (.907). However, the adjusted goodness-of-fit index (AGFI, acceptable value $>.90$ ), Tucker and Lewis's non-normed fit index (TLI, acceptable value $>.90$ ) were also found to be unacceptable: .511 and .150 respectively, for this model. The ratio of Maximum-Likelihood Chi-Square to the degrees of freedom $\left(\mathrm{X}^{2} / \mathrm{df}\right.$, acceptable value $\left.<5\right)$ was also found to be unacceptable 20.62.

In order to develop a better-fitting model, some Post Hoc Model modifications were conducted successively. At first, Guilt was allowed to predict Attribution and further, the error variances of Realization \& Reparation and Guilt, as well as Positive and Negative Self-compassion, was made correlated. However, to obtain a perfect fit model one more modification was done by removing the insignificant predictive pathway from the model. The pathway leading from Self-Esteem to Guilt was removed subsequently. Only a single modification was adapted at a time. These modifications produced a perfect fit model: $\mathrm{X}^{2} / \mathrm{df}(\mathrm{df}=2)=1.566$; $\mathrm{CFI}=.996 ; \mathrm{RMSEA}=.04 ; \mathrm{SRMR}=.017 ; \mathrm{GFI}=.996, \mathrm{AGFI}=.959$ (Figure 2).

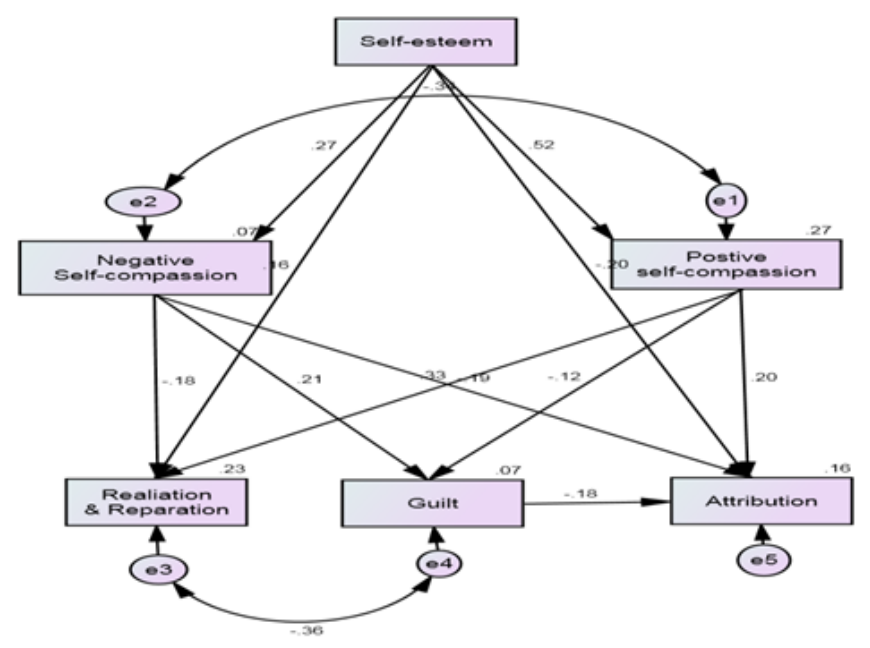

Figure 2: Figure 2 (Revised Model) A path model taking positive and negative self-compassion as mediators in the relationship between self-esteem and self-forgiveness depicting (realization \& reparation, guilt \& attribution).

The standardized direct path coefficients of the tested model (figure 2) are presented in table 2.

Table 2. Direct effects of self-esteem and self-compassion on the dimensions of self-forgiveness

Estimate

Negative self-compassion

$<-$

Self esteem

$.27^{* *}$

Positive self-compassion 
$<-$

Self esteem

$.52^{* *}$

Guilt

$<-$

Negative self-compassion $.21^{* * *}$

Guilt

$<-$

Positive self-compassion

$-.12^{*}$

Attribution

$<-$

Self esteem

$-.21^{*}$

Attribution

$<-$

Negative self-compassion

$-.19^{* * *}$

Realization \& reparation

$<-$

Positive self-compassion $.34^{*}$

Attribution

$<-$

Positive self-compassion $.20^{*}$

Realization \& reparation $<-$

Self esteem

$.16^{*}$

Realization \& reparation $<-$

Negative self-compassion 
$-.18 * * *$

Attribution

$<-$

Guilt

$-.183$

${ }^{*} * \mathrm{p}<.01,{ }^{*} \mathrm{p}<.05$

It was found that self-esteem directly predicted both positive and negative self-compassion (path coefficients $.52 \& .27$ respectively, $\mathrm{p}<.01$ ). Self-esteem significantly and directly predicted realization \&reparation as well as attribution (path coefficients $.16 \&-.21$ respectively, $\mathrm{p}<.05$ ). Negative self-compassion significantly predicted all the dimensions of self-forgiveness viz. realization \& reparation, guilt and attribution (path coefficients -.18, .21 \& -.19 respectively, $\mathrm{p}<.01)$. Positive self-compassion significantly predicted realization \& reparation, guilt as well as attribution (path coefficients .34, -.12\& .20 respectively, p $<.05$ ).

The indirect (mediating) effects of Self-Esteem on the dimensions of Self-Forgiveness were calculated and checked for statistical significance. The significant indirect paths are presented in table 3 .

Table 3. Indirect effects of self-esteem on the dimensions of self-forgiveness

\begin{tabular}{ll}
\hline Indirect effects of self-Esteem on realization & Estimate \\
\&reparation & $-.05^{* *}$ \\
Through Negative Self-Compassion & $.18^{* *}$ \\
Through Positive Self-Compassion & .125 \\
Total & \\
Indirect effects of self-esteem on guilt & $.06^{* *}$ \\
Through Negative Self-Compassion & $-.06^{* *}$ \\
Through Positive Self-Compassion & 00 \\
Total & \\
Indirect effects of self-esteem on attribution & $-.05^{* *}$ \\
Through Negative Self-Compassion & $.11^{*}$ \\
Through Positive Self-Compassion & .06 \\
Total & \\
\hline
\end{tabular}

$$
{ }^{*} \mathrm{p}<.01,{ }^{*} \mathrm{p}<.05
$$

It was found that Self-esteem had dual and opposing indirect effects on the dimensions of Self-forgiveness. Both Negative and Positive Self-Compassion significantly mediated the relationship between Self-Esteem and Self-Forgiveness dimensions but due to their opposite directionality, the total mediating effects became non-significant (Table 3).

\section{Discussion}

The findings of the study have evinced the significant role of Self-Esteem and Self-Compassion in impacting the nature and extent of Self-Forgiveness of the participants. Self-Esteem, Positive and Negative SelfCompassion have shown their dissimilar contributions to the three components of Self-Forgiveness. Positive and negative self-compassion significantly mediated the relationships among self-esteem and the three components of self-forgiveness (Realization \& Reparation, guilt and attribution). 


\section{Self-Esteem and Self-Forgiveness}

The findings showed that Self-Esteem exhibited significant positive correlations with Realization \& Reparation and overall self-forgiveness while significant negative or very low positive correlations with Guilt and Attribution. The hierarchical regression analysis showed that Self-Esteem accounted for significant variance in the scores of Realization \& Reparation and emerged as the most significant predictor next to Positive and Negative Self-compassion. Likewise, Self-Esteem accounted for significant variance in the scores of guilt and Attribution. In essence, Self-Esteem emerged as an important contributor to Realization \&Reparation after Positive Self-Compassion. The findings showed that Self-Esteem has inhibiting effects on Attribution. These findings partially support hypotheses 1,2 and 3.

The mechanisms underlying the role of self-esteem in impacting self-forgiveness can be understood and explained in terms of the basic properties of these constructs. Self-esteem carries self-satisfaction, selfattitude, self-worth, self-efficacy, self-respect, social comparison, self-pride, self-perception, relative strengths and feeling of being virtuous (Pandey et al., 2019; Rosenberg, 1965). Self-esteem has been reported to have close links with happiness, positive emotions, self-evaluation, positive mood regulation, optimistic thinking and optimal functioning that buffer the effects of stress caused by pain, failure and adversities of life. It has also been observed that self-esteem benefits individuals in two ways: enhanced initiative and pleasant feelings (Baumeister et al., 2003; Pandey et al., 2019).

Self-forgiveness measure used in this study has three components: realization \& reparation, guilt and attribution. Realization \& reparation involves a feeling of wrongdoing, taking responsibility and easily accepting wrongdoings, repairing of relationship, tendency and ability to minimize negative emotions to self, promise to not repeat wrongdoing again, others good behaviours, remorse, forgetting of past wrongdoing, forgiveness to others, the importance of relationships, self-love, readiness to correct, seeking forgiveness, positive behaviour towards others, degree of loss, physical and emotional consequences, time, assessing situational responsibility and morality (Mudgal \& Tiwari, 2017a). Guilt involves self-criticism, accepting the responsibility of transgression and its consequences (Mudgal \& Tiwari, 2017a). Attribution comprises inclination for improvement, nature and relationship, seeking forgiveness to others, perception of social relationships, moral values and self-criticism (Mudgal \& Tiwari, 2017a).

These affirmative attributes of self-esteem might be playing a causative role behind its close links with realization \& reparation, attribution and overall self-forgiveness of the participants in this study. The findings of the present study lend their support from previous studies. For example, a good number of studies have suggested that self-esteem plays a promotive role in self-forgiveness and the individuals with the threat to self-esteem have a higher need for self-protection (Leary et al., 2009; vanDellen et al., 2011) and, thus, low self-esteem is associated with a lower level of forgiveness. It has been suggested that individuals with high self-esteem are better able to deflect threats against the self (Pyszczynski et al., 2004). Moreover, people with lower self-esteem are absorbed in protecting the self and lack required self-resources to invest in self-forgiveness while people with higher self-esteem have sufficient self-resources necessary for self-forgiveness (Baumeister et al., 2007).

\section{Self-Compassion and Self-Forgiveness}

The findings pertaining to the relationship between self-compassion and self-forgiveness are very insightful. There were significant positive correlations among the scores of positive and negative self-compassion and the scores of realization \& reparation, attribution and overall self-forgiveness of the participants. Conversely, significant negative correlations were observed among the scores of positive self-compassion and the scores of guilt and attribution. Moreover, negative self-compassion had shown significant negative correlations with the scores of realization \& reparation, guilt, attribution and overall self-forgiveness. The hierarchical regression analysis showed that positive and negative self-compassion accounted for significant variance in

the scores of realization \& reparation, attribution and overall self-forgiveness and the former emerged as the most significant predictor of these measures. Positive and negative self-compassion accounted for significant 
variance in the scores of guilt also and negative self-compassion emerged as the most significant predictor of guilt. Positive self-compassion has shown enhancing role in shaping the nature and extent of self-forgiveness while negative self-compassion had a diminishing impact on self-forgiveness. Interestingly, both Positive and Negative Self-Compassion significantly mediated the relationship between Self-Esteem and Realization \& Reparation, Guilt and Attribution. These findings support hypotheses 1, 2, 3 and 4.

The findings of the study demonstrated that due to their dissimilar nature, positive and negative self-compassion differentially mediated the relationships among self-esteem and the three components of selfforgiveness (realization \& reparation, guilt and attribution). This may be explained in two ways. In one way, the dissimilar nature of positive and negative self-compassion may be applied to understand their impacts on self-forgiveness while the second may involve the mechanisms reported by earlier findings. In essence, positive self-compassion comprises self-kindness, common humanity and mindfulness that reflect self-acceptance, selfcare, self-kindness, self-tolerance, understanding and patience towards negative self-traits, common struggle, shared perception about lack of resources, shared/common inadequacy, emotional stability, stable perception, analytical thinking about failure in important domains of life and open-mindedness towards self during hurtful times (Neff, 2003b). On the other hand, negative self-compassion consists of self-judgment, isolation and over-identification that carry critical attitude towards self, harsh treatment towards self, intolerance towards negative traits of self, self-depreciation, harsh treatment, feeling of loneliness, self-comparison, thinking about others, feeling of loneliness during failure in important domains of life, indulgence in negative habits, feeling of inferiority and over-identification and maximization of events (Neff, 2003b). Thus, the debilitating tendency of negative self-compassion might be working behind its close association with guilt and its negative correlation and lowered predictive strength for other aspects of self-forgiveness. These attributes of positive and negative self-compassion may be assumed to underlie their dissimilar impacts for realization \& reparation, guilt and attribution as well as overall self-forgiveness.

The earlier researchers have also suggested that self-compassion and self-forgiveness are very closely linked as both occur simultaneously, reflect self-knowledge, self-attitude and have similar positive impacts on the individuals. The recent bifurcation of self-compassion in positive and negative dimensions has empirical implications to understand and explain performance and outcomes (Muris et al., 2016; Pandey et al., 2019). It has been reported that positive self-compassion has a negative association with mental health problems and psychopathological symptoms whereas negative self-compassion carries a positive association with these symptoms (Muris \& Petrocchi, 2017). The differences in the predictive strengths of positive and negative self-compassion may lie in their dissimilar attributes and mechanisms for various life outcomes.

The followers of two components model of self-compassion argue that positive and negative self-compassion reflects two distinct mechanisms which have been empirically verified (Muris et al., 2018; Pandey et al., 2019). It has been reported that self-judgment, isolation and over-identification exhibit clear similarities with harsh self-criticism (Zuroff et al., 1990), social withdrawal and loneliness (Rubin \& Coplan, 2004), and self-absorption and self-focused rumination (Lyubomirsky \& Nolen-Hoeksema, 1995). Positive self-compassion has been reported to be linked with adaptive coping and healthy functioning while negative self-compassion with maladaptive coping, anxiety and depression (Muris et al., 2018). It has been suggested that positive self-compassion and various adaptive coping styles are positively correlated while negative self-compassion showed a negative association with these variables (Allen \& Leary, 2010). Negative self-compassion has been reported to reflect passive reacting, avoidance, and expression of emotion (Allen \& Leary, 2010). The findings of the study suggest that conceptualization of self-compassion in terms of positive and negative dimensions are more efficacious to underscore its role in self-forgiveness.

The distinctive role of self-esteem and self-compassion in shaping the self-forgiveness may be understood in terms of their conceptualizations. Research indicates that self-compassion is moderately associated with trait levels of self-esteem (Leary et al., 2007; Neff, 2003a; Neff et al., 2007). Self-esteem refers to the degree to which people evaluate and like or value themselves in comparisons to others (Harter, 1999). In other words, high self-esteem means standing out in a crowd, being special and above average (Heine et al., 1999). Conversely, self-compassion is not based on positive or negative evaluations and reflects 
a way to relate one with oneself. Self-compassion occurs not due to relative evaluation but it happens as people are human beings (Neff, 2012). Moreover, self-compassion comprises being warm and understanding in the face of suffering and adversity (Neff, 2012).

Self-esteem and self-compassion differ also in the benefits they provide to the people who carry. For example, self-compassion facilitates personal adequacy, care, acknowledgement of problems, common experience, receptive mind-state, identification with feelings and thoughts (Bishop et al., 2006). It emphasizes interconnection rather than separateness. In addition, self-compassion offers more emotional stability, connectedness, acceptance, autonomy, greater relationship satisfaction and attachment security as well as less detached, controlling, and verbally or physically aggressive than those lacking self-compassion (Neff, 2012). Self-esteem benefits people in other ways. For example, people with high self-esteem exhibit lessened depression and anxiety (McKay \& Fanning, 2016).

The above discussion makes it apparent that both self-esteem and self-compassion are desirable attributes and differ in their mechanisms that may be assumed to underlie their dissimilar roles in selfforgiveness. In essence, self-compassion is an emotionally positive self-attitude that has a close connection with self-esteem (Neff, 2003a, 2011)and helps the individuals to respond adaptively to the negative experiences and threats and overlaps with self-esteem. Self-esteem is assumed to be close to self-affirmation as the later involves balanced and open-minded appraisal (Burson et al., 2012). The above discussion makes it clear that self-esteem and self-compassion carry significance to explicate the nature and dynamics of self-forgiveness of the individuals.

In short, the findings showed that both self-esteem and self-compassion carried their significance to understand self-forgiveness of the participants. Self-Esteem and Positive Self-Compassion had evinced significant positive correlations with Self-Forgiveness. Conversely, Negative Self-Compassion exhibited negative correlations with these measures. Self-Esteem accounted for significant positive variance in Realization 85 Reparation and Overall Self-Forgiveness and significant negative variability in Attribution of the participants. Positive Self-Compassion accounted for positive significant variance in Realization $\&$ Reparation and Overall SelfForgiveness whereas negative variance in Guilt and Attribution. On the other hand, Negative Self-Compassion accounted for positive significant variance in Guilt and negative significant variability in Realization ESReparation and Attribution. It was evident that both Positive and Negative Self-Compassion significantly mediated the relationship between Self-Esteem and Realization \& Reparation, Guilt and Attribution.

The researchers have argued self-esteem is more relevant to understand the performance and life outcomes of people belonging to individualistic societies (Fiske et al., 1998; Triandis, 1989, 2001)whereas selfcompassion may be more efficacious to understand the performance of individuals brought up in collectivistic societies (Birkett, 2013; Kitayama \& Uskul, 2011; Markus \& Kitayama, 2010; Neff et al., 2008). Contrarily, here both the constructs have shown their relevance to understand self-forgiveness. This may due to the fact that both self-esteem and self-compassion may be working together as has been suggested by the researchers that individualism and collectivism may coexist in the societies like India (Pandey et al., 2019; Sinha 83 Tripathi, 1994). Likewise, both independent and interdependent self-affirmations have been reported to be active after the outbreak of COVID-19 in recent Indian studies (Pandey et al., 2020; Tiwari et al., 2020). Thus, these findings may represent the truth that both self-esteem and self-compassion were present and regulated performance on a positive life outcome measure (self-forgiveness). In essence, the findings may be indicative of the fact that Indian society is in transition that is reflected by the existence of self-esteem and self-compassion shaping positively the self-forgiveness of the participants in the study. The findings may prompt future researchers to have a fresh look at many other psychological constructs that have been seen the individual and collectivism dichotomy. It proved the contention that qualitative studies may be conducted to explore the true nature of many psychological constructs to come up with new understanding and measures for the psychological constructs. 


\section{References}

Allen, A. B., Goldwasser, E. R., \& Leary, M. R. (2012). Self-compassion and Well-being among Older Adults. Self and Identity, 11(4), 428-453. https://doi.org/10.1080/15298868.2011.595082

Allen, A. B., \& Leary, M. R. (2010). Self-Compassion, Stress, and Coping. Social and Personality Psychology Compass, 4(2), 107-118. https://doi.org/10.1111/j.1751-9004.2009.00246.x

Aquino, K., Tripp, T. M., \& Bies, R. J. (2006). Getting even or moving on? Power, procedural justice, and types of offense as predictors of revenge, forgiveness, reconciliation, and avoidance in organizations. Journal of Applied Psychology, 91 (3), 653-668. https://doi.org/10.1037/0021-9010.91.3.653

Arimitsu, K. (2014). Development and validation of the Japanese version of the Self-Compassion Scale. Shinrigaku Kenkyu: The Japanese Journal of Psychology, 85(1), 50-59. https://doi.org/10.4992/jjpsy.85.50

Azizi, A., Mohammadkhani, P., Foroughi, A. akbar, Lotfi, S., \& Bahramkhani, M. (2013). The Validity and Reliability of the Iranian Version of the Self-Compassion Scale. Practice in Clinical Psychology, 1(3), $149-155$.

Baskin, T. W., \& Enright, R. D. (2004). Intervention Studies on Forgiveness: A Meta-Analysis. Journal of Counseling \& Development, 82(1), 79-90. https://doi.org/10.1002/j.1556-6678.2004.tb00288.x

Baumeister, R. F., Campbell, J. D., Krueger, J. I., \& Vohs, K. D. (2003). Does High Self-Esteem Cause Better Performance, Interpersonal Success, Happiness, or Healthier Lifestyles? Psychological Science in the Public Interest, 4 (1), 1-44. https://doi.org/10.1111/1529-1006.01431

Baumeister, R. F., Vohs, K. D., \& Tice, D. M. (2007). The Strength Model of Self-Control. Current Directions in Psychological Science, 16(6), 351-355. https://doi.org/10.1111/j.1467-8721.2007.00534.x

Berry, J. W., Worthington, E. L., Wade, N. G., van Oyen Witvliet, C., \& Kiefer, R. P. (2005). FORGIVENESS, MORAL IDENTITY, AND PERCEIVED JUSTICE IN CRIME VICTIMS AND THEIR SUPPORTERS. Humboldt Journal of Social Relations, 29(2), 136-162. JSTOR.

Birkett, M. A. (2013). Self-compassion and empathy across cultures: Comparison of young adults in China and the United States. International Journal of Research Studies in Psychology, 3(1). https://doi.org/10.5861/ijrsp.2013.551

Biron, D. E. (2007). Personality, substance abuse, and lack of self-forgiveness with incarcerated males ((67), ProQuest Information \& Learning, US). ProQuest Information \& Learning, US; ProQuest Information \& Learning, US. http://search.ebscohost.com/login.aspx?direct $=$ true \&db=psyh\&AN=2007-99010-087\& site $=$ ehost-live Available from EBSCOhost psyh database

Bishop, S. R., Lau, M., Shapiro, S., Carlson, L., Anderson, N. D., Carmody, J., Segal, Z. V., Abbey, S., Speca, M., Velting, D., \& Devins, G. (2006). Mindfulness: A Proposed Operational Definition. Clinical Psychology: Science and Practice, 11(3), 230-241. https://doi.org/10.1093/clipsy.bph077

Burson, A., Crocker, J., \& Mischkowski, D. (2012). Two Types of Value-Affirmation: Implications for Self-Control Following Social Exclusion. Social Psychological and Personality Science, 3(4), 510-516. https://doi.org/10.1177/1948550611427773

Butzen, N. D. (2009). NEO Personality Inventory-Revised five-factor model facets as predictors of forgiveness. ProQuest Information \& Learning, US. http://search.ebscohost.com/login.aspx?direct $=$ true $\& d b=$ psyh \&AN $=2009-99120-\quad$ 058\&site $=$ ehost-live Available from EBSCOhostpsyh database.

Castilho, P., Pinto-Gouveia, J., \& Duarte, J. (2015). Evaluating the Multifactor Structure of the Long and Short Versions of the Self-Compassion Scale in a Clinical Sample: Factor Analysis of the Long and Short Self-Compassion Scale. Journal of Clinical Psychology, 71(9), 856-870. https://doi.org/10.1002/jclp.22187 
Christensen, K. J., Padilla-Walker, L. M., Busby, D. M., Hardy, S. A., \& Day, R. D. (2011). Relational and social-cognitive correlates of early adolescents' forgiveness of parents. Journal of Adolescence, 34(5), 903-913. https://doi.org/10.1016/j.adolescence.2011.01.001

Cleare, S., Gumley, A., Cleare, C. J., \& O'Connor, R. C. (2018). An Investigation of the Factor Structure of the Self-Compassion Scale. Mindfulness, 9(2), 618-628. https://doi.org/10.1007/s12671-017-0803-1

Coates, D. (1996). The Correlations of Forgiveness of Self, Forgiveness of Others, and Hostility, Depression, Anxiety, Self-esteem, Life Adaptation, and Religiosity Among Female Victims of Domestic Violence. California School of Professional Psychology, Fresno.

Conway-Williams, E. (2015). Self-Compassion and Self-Forgiveness as Mediated by Rumination, ShameProneness, and Experiential Avoidance: Implications for Mental and Physical Health. Electronic Theses and Dissertations. https://dc.etsu.edu/etd/2562

Donald, S. (2016). Data Screening using SPSS for beginner: Outliers, Missing Values and Normality [Workshop]. Institute of Borneo Studies Workshop Series 2016 (2)3, Institute of Borneo Studies, UNIMAS. https://ir.unimas.my/id/eprint/12266/

Enright, R. D. (1996). Counseling Within the Forgiveness Triad: On Forgiving, Receiving Forgiveness, and Self-Forgiveness. Counseling and Values, 40(2), 107-126. https://doi.org/10.1002/j.2161007X.1996.tb00844.x

Fehr, R., Gelfand, M. J., \& Nag, M. (2010). The road to forgiveness: A meta-analytic synthesis of its situational and dispositional correlates. Psychological Bulletin, 136(5), 894-914. https://doi.org/10.1037/a0019993

Fincham, F. D., Jackson, H., \& Beach, S. R. H. (2005). Transgression Severity and Forgiveness: Different Moderators for Objective and Subjective Severity. Journal of Social and Clinical Psychology, 24 (6), 860-875. https://doi.org/10.1521/jscp.2005.24.6.860

Fiske, A. P., Kitayama, S., Markus, H. R., \& Nisbett, R. E. (1998). The cultural matrix of social psychology. In The Handbook of Social Psychology, Vol. 2, eds D. T. Gilbert, S. T. Fiske, and G. Lindzey (pp. 915-981). Wiley.

Garcia-Campayo, J., Navarro-Gil, M., Andrés, E., Montero-Marin, J., López-Artal, L., \& Demarzo, M. M. (2014). Validation of the Spanish versions of the long (26 items) and short (12 items) forms of the Self-Compassion Scale (SCS). Health and Quality of Life Outcomes, 12(1), 4. https://doi.org/10.1186/1477$7525-12-4$

Griffin, B. J., Cowden, R. G., \& Shawkey, L. A. (2020). Bibliography of narrative reviews and meta-analyses on forgiveness (2004-2018). In E. L., Worthington, Jr., 83 N. G., Wade, (Eds.), Handbook of forgiveness (Second Edition, pp. 335-344). Routledge.

Hall, J. H., \& Fincham, F. D. (2005). Self-Forgiveness: The Stepchild of Forgiveness Research. Journal of Social and Clinical Psychology, 24(5), 621-637. https://doi.org/10.1521/jscp.2005.24.5.621

Hall, J. H., \& Fincham, F. D. (2008). The Temporal Course of Self-Forgiveness. Journal of Social and Clinical Psychology, 27(2), 174-202. https://doi.org/10.1521/jscp.2008.27.2.174

Harter, S. (1999). The construction of the self: A developmental perspective. Guilford Press.

Heine, S. J., Lehman, D. R., Markus, H. R., \& Kitayama, S. (1999). Is there a universal need for positive self-regard? Psychological Review, 106(4), 766-794. https://doi.org/10.1037/0033-295X.106.4.766

Hirsch, J. K., Webb, J. R., \& Jeglic, E. L. (2011). Forgiveness, depression, and suicidal behavior among a diverse sample of college students. Journal of Clinical Psychology, 67(9), 896-906. https://doi.org/10.1002/jclp.20812 
Hu, L., \& Bentler, P. M. (1999). Cutoff criteria for fit indexes in covariance structure analysis: Conventional criteria versus new alternatives. Structural Equation Modeling: A Multidisciplinary Journal, 6(1), 1-55. https://doi.org/10.1080/10705519909540118

Jöreskog, K. G., \& Sörbom, D. (1998). LISREL 8: Structural equation modeling with the SIMPLIS command language. Erlbaum.

Kitayama, S., \& Uskul, A. K. (2011). Culture, Mind, and the Brain: Current Evidence and Future Directions. Annual Review of Psychology, 62(1), 419-449. https://doi.org/10.1146/annurev-psych-120709-145357

Lawler, K. A., Younger, J. W., Piferi, R. L., Billington, E., Jobe, R., Edmondson, K., \& Jones, W. H. (2003). A change of heart: Cardiovascular correlates of forgiveness in response to interpersonal conflict. Journal of Behavioral Medicine, 26(5), 373-393.

Leary, M. R., Tate, E. B., Adams, C. E., Allen, A. B., \& Hancock, J. (2007). Self-compassion and reactions to unpleasant self-relevant events: The implications of treating oneself kindly. Journal of Personality and Social Psychology, 92(5), 887-904. https://doi.org/10.1037/0022-3514.92.5.887

Leary, M. R., Terry, M. L., Batts Allen, A., \& Tate, E. B. (2009). The concept of ego threat in social and personality psychology: Is ego threat a viable scientific construct? Personality and Social Psychology Review: An Official Journal of the Society for Personality and Social Psychology, Inc, 13(3), 151-164. https://doi.org/10.1177/1088868309342595

López, A., Sanderman, R., Smink, A., Zhang, Y., van Sonderen, E., Ranchor, A., \& Schroevers, M. J. (2015). A Reconsideration of the Self-Compassion Scale's Total Score: Self-Compassion versus Self-Criticism. PLOS ONE, 10(7), e0132940. https://doi.org/10.1371/journal.pone.0132940

Lyubomirsky, S., \& Nolen-Hoeksema, S. (1995). Effects of self-focused rumination on negative thinking and interpersonal problem solving. Journal of Personality and Social Psychology, 69(1), 176-190. https://doi.org/10.1037//0022-3514.69.1.176

MacBeth, A., \& Gumley, A. (2012). Exploring compassion: A meta-analysis of the association between self-compassion and psychopathology. Clinical Psychology Review, 32(6), 545-552. https://doi.org/10.1016/j.cpr.2012.06.003

MacKinnon, N. J. (2015). Self-Esteem and Beyond. Palgrave Macmillan UK. https://doi.org/10.1057/9781137542304

Maltby, J., Macaskill, A., \& Day, L. (2001). Failure to forgive self and others: A replication and extension of the relationship between forgiveness, personality, social desirability and general health. Personality and Individual Differences, 30(5), 881-885. https://doi.org/10.1016/S0191-8869(00)00080-5

Markus, H. R., \& Kitayama, S. (2010). Cultures and Selves: A Cycle of Mutual Constitution. Perspectives on Psychological Science, 5(4), 420-430. https://doi.org/10.1177/1745691610375557

McConnell, J. M., Dixon, D. N., \& Finch, W. H. (2012). An Alternative Model of Self-Forgiveness. The New School Psychology Bulletin, 9(2), 35-51.

McCullough, M. E. (2008). Beyond revenge: The evolution of the forgiveness instinct (1st ed). Jossey-Bass.

McCullough, M. E., Bono, G., \& Root, L. M. (2007). Rumination, emotion, and forgiveness: Three longitudinal studies. Journal of Personality and Social Psychology, 92(3), 490-505. https://doi.org/10.1037/00223514.92.3.490

McCullough, M. E., Rachal, K. C., Sandage, S. J., Worthington, E. L., Brown, S. W., \& Hight, T. L. (1998). Interpersonal forgiving in close relationships: II. Theoretical elaboration and measurement. Journal of Personality and Social Psychology, 75 (6), 1586-1603. https://doi.org/10.1037/0022-3514.75.6.1586 
McGaffin, B. J., Lyons, G. C. B., \& Deane, F. P. (2013). Self-Forgiveness, Shame, and Guilt in Recovery from Drug and Alcohol Problems. Substance Abuse, 34(4), 396-404. https://doi.org/10.1080/08897077.2013.781564

McKay, M., \& Fanning, P. (2016). Self-esteem: A proven program of cognitive techniques for assessing, improving, $\mathcal{E}$ maintaining your self-esteem (Fourth edition). New Harbinger Publications, Inc.

McNulty, J. K. (2011). The Dark Side of Forgiveness: The Tendency to Forgive Predicts Continued Psychological and Physical Aggression in Marriage. Personality and Social Psychology Bulletin, 37(6), 770-783. https://doi.org/10.1177/0146167211407077

Mudgal, S., \& Tiwari, G. K. (2017a). The Self-forgiveness Scale. Unpublished Scale, Department of Psychology, School of Humanities \& Social Sciences, Doctor Harisingh Gour Vishwavidyalaya, Sagar, Madhya Pradesh, India.

Mudgal, S., \& Tiwari, G. K. (2017b). High level of self-forgiveness facilitates quality of life in males and females. Vaichariki-A Multidisciplinary Refereed International Research Journal, 8(3), 154-166.

Muris, P., Otgaar, H., \& Petrocchi, N. (2016). Protection as the Mirror Image of Psychopathology: Further Critical Notes on the Self-Compassion Scale. Mindfulness, 7(3), 787-790. https://doi.org/10.1007/s12671016-0509-9

Muris, P., \& Petrocchi, N. (2017). Protection or Vulnerability? A Meta-Analysis of the Relations Between the Positive and Negative Components of Self-Compassion and Psychopathology. Clinical Psychology \&5 Psychotherapy, 24 (2), 373-383. https://doi.org/10.1002/cpp.2005

Muris, P., van den Broek, M., Otgaar, H., Oudenhoven, I., \& Lennartz, J. (2018). Good and Bad Sides of Self-Compassion: A Face Validity Check of the Self-Compassion Scale and an Investigation of its Relations to Coping and Emotional Symptoms in Non-Clinical Adolescents. Journal of Child and Family Studies, 27(8), 2411-2421. https://doi.org/10.1007/s10826-018-1099-z

Neff, K. D. (2003a). Self-Compassion: An Alternative Conceptualization of a Healthy Attitude Toward Oneself. Self and Identity, 2(2), 85-101. https://doi.org/10.1080/15298860309032

Neff, K. D. (2003b). The Development and Validation of a Scale to Measure Self-Compassion. Self and Identity, 2(3), 223-250. https://doi.org/10.1080/15298860309027

Neff, K. D. (2011). Self-Compassion, Self-Esteem, and Well-Being: Self-Compassion, Self-Esteem, and Well-Being. Social and Personality Psychology Compass, 5(1), 1-12. https://doi.org/10.1111/j.17519004.2010.00330.x

Neff, K. D. (2012). The science of self-compassion. In C. Germer $\mathcal{E}$ R. Siegel (eds.), Compassion and Wisdom in Psychotherapy (pp. 79-92). Guilford Press.

Neff, K. D., Pisitsungkagarn, K., \& Hsieh, Y.-P. (2008). Self-Compassion and Self-Construal in the United States, Thailand, and Taiwan. Journal of Cross-Cultural Psychology, 39(3), 267-285. https://doi.org/10.1177/0022022108314544

Neff, K. D., \& Pommier, E. (2013). The Relationship between Self-compassion and Other-focused Concern among College Undergraduates, Community Adults, and Practicing Meditators. Self and Identity, 12(2), 160-176. https://doi.org/10.1080/15298868.2011.649546

Neff, K. D., Rude, S. S., \& Kirkpatrick, K. L. (2007). An examination of self-compassion in relation to positive psychological functioning and personality traits. Journal of Research in Personality, 41(4), 908916. https://doi.org/10.1016/j.jrp.2006.08.002

Neff, K. D., Whittaker, T. A., \& Karl, A. (2017). Examining the Factor Structure of the Self-Compassion Scale in Four Distinct Populations: Is the Use of a Total Scale Score Justified? Journal of Personality Assessment, 99(6), 596-607. https://doi.org/10.1080/00223891.2016.1269334 
Noor, M., Brown, R., Gonzalez, R., Manzi, J., \& Lewis, C. A. (2008). On Positive Psychological Outcomes: What Helps Groups With a History of Conflict to Forgive and Reconcile With Each Other? Personality and Social Psychology Bulletin, 34(6), 819-832. https://doi.org/10.1177/0146167208315555

Pandey, R., Tiwari, G. K., Parihar, P., \& Rai, P. K. (2019). Positive, not negative, self-compassion mediates the relationship between self-esteem and well-being. Psychology and Psychotherapy: Theory, Research and Practice, papt.12259. https://doi.org/10.1111/papt.12259

Pandey, R., Tiwari, G. K., Parihar, P., \& Rai, P. K. (2020). The relationship between self-forgiveness and human flourishing: Inferring the underlying psychological mechanisms. Polish Psychological Bulletin, 51 (1), 29-42. https://doi.org/10.24425/ppb.2020.132649

Pandey, R., Tiwari, G. K., \& Rai, P. K. (2020). The Independent and Interdependent Selfaffirmations in Action: Understanding their dynamics in India during COVID-19 [Preprint]. Preprints. https://doi.org/10.22541/au.158958018.83880224

Pfattheicher, S., Geiger, M., Hartung, J., Weiss, S., \& Schindler, S. (2017). Old Wine in New Bottles? The Case of Self-compassion and Neuroticism: Self-compassion and neuroticism. European Journal of Personality, 31(2), 160-169. https://doi.org/10.1002/per.2097

Pyszczynski, T., Greenberg, J., Solomon, S., Arndt, J., \& Schimel, J. (2004). Why Do People Need Self-Esteem? A Theoretical and Empirical Review. Psychological Bulletin, 130(3), 435-468. https://doi.org/10.1037/0033-2909.130.3.435

Rai, P. K., \& Tiwari, G. K. (2019). Self-Compassion and Positive Mental Health. In S. Ojha, M. Asthana, \& U. Ojha, Spirituality 83 health: Emerging issues (pp. 175-201). Shree Publishers \& Distributors.

Rangganadhan, A. R., \& Todorov, N. (2010). Personality and Self-Forgiveness: The Roles of Shame, Guilt, Empathy and Conciliatory Behavior. Journal of Social and Clinical Psychology, 29(1), 1-22. https://doi.org/10.1521/jscp.2010.29.1.1

Rosenberg, M. (1965). Society and the adolescent self-image. Princeton University Press.

Rubin, K. H., \& Coplan, R. J. (2004). Paying Attention to and Not Neglecting Social Withdrawal and Social Isolation. Merrill-Palmer Quarterly, 50(4), 506-534. https://doi.org/10.1353/mpq.2004.0036

Scherer, M., Worthington, E. L., Hook, J. N., \& Campana, K. L. (2011). Forgiveness and the Bottle: Promoting Self-Forgiveness in Individuals Who Abuse Alcohol. Journal of Addictive Diseases, 30(4), 382395. https://doi.org/10.1080/10550887.2011.609804

Sinha, D., \& Tripathi, R. C. (1994). Individualism in a collectivist culture: A case of coexistence of opposites. In U. Kim, H. C. Triandis, C. Kağitçibaşi, S. C. Choi, \& G. Yoon (Eds.), Cross-cultural research and methodology series, Vol. 18. Individualism and collectivism: Theory, method, and applications (pp. 123136). Sage Publications.

Sirois, F. M., Molnar, D. S., \& Hirsch, J. K. (2015). Self-Compassion, Stress, and Coping in the Context of Chronic Illness. Self and Identity, 14 (3), 334-347. https://doi.org/10.1080/15298868.2014.996249

Steele, C. M., Spencer, S. J., \& Lynch, M. (1993). Self-image resilience and dissonance: The role of affirmational resources. Journal of Personality and Social Psychology, 64(6), 885-896. https://doi.org/10.1037//0022-3514.64.6.885

Strelan, P., \& Zdaniuk, A. (2015). Threatened State Self-Esteem Reduces Forgiveness. Self and Identity, 14(1), 16-32. https://doi.org/10.1080/15298868.2014.889034

Thompson, L. Y., Snyder, C. R., Hoffman, L., Michael, S. T., Rasmussen, H. N., Billings, L. S., Heinze, L., Neufeld, J. E., Shorey, H. S., \& Roberts, J. C. (2005). Dispositional Forgiveness of Self, Others, and Situations. JOURNAL OF PERSONALITY, 73(2), 313-360. 
Tiwari, G. K., Kashyap, A. K., Rai, P. K., Tiwari, R. P., \& Pandey, R. (2020). The Collective-affirmation in Action: Understanding the Success of Lockdown in India after the Outbreak of COVID-19 [Preprint]. Preprints. https://doi.org/10.22541/au.158949202.27402247

Toussaint, L. L., \& Webb, J. R. (2005). Theoretical and empirical connections between forgiveness, mental health, and well-being. In E. L. J. Worthington (Ed.), Handbook of forgiveness (pp. 349-362). Routledge.

Toussaint, L. L., Worthington Jr, E. L., Williams, D. R., \& Webb, J. R. (2020). Forgiveness and Physical Health. In E. L. Worthington Jr. \& N. G. Wade (Ed.), Handbook of Forgiveness (Second Edition, pp. 178-187). Routledge.

Triandis, H. C. (1989). The self and social behavior in differing cultural contexts. Psychological Review, 96 (3), 506-520. https://doi.org/10.1037/0033-295X.96.3.506

Triandis, H. C. (2001). Individualism-Collectivism and Personality. Journal of Personality, 69(6), 907-924. https://doi.org/10.1111/1467-6494.696169

vanDellen, M. R., Campbell, W. K., Hoyle, R. H., \& Bradfield, E. K. (2011). Compensating, Resisting, and Breaking: A Meta-Analytic Examination of Reactions to Self-Esteem Threat. Personality and Social Psychology Review, 15(1), 51-74. https://doi.org/10.1177/1088868310372950

Verma, Y., \& Tiwari, G. K. (2017). Self-Compassion as the Predictor of Flourishing of the Students. The International Journal of Indian Psychology, 4(3), 10-29. https://doi.org/10.25215/0403.122

Webb, J. R., \& Toussaint, L. L. (2020). Forgiveness, Well-Being, and Mental Health. In E. L., Worthington, Jr., 83 N. G., Wade, (Eds.), Handbook of forgiveness (Second Edition, pp. 188-197). Routledge.

Webb, Jon R., Hirsch, J. K., Visser, P. L., \& Brewer, K. G. (2013). Forgiveness and Health: Assessing the Mediating Effect of Health Behavior, Social Support, and Interpersonal Functioning. The Journal of Psychology, 147(5), 391-414. https://doi.org/10.1080/00223980.2012.700964

Wenzel, M., Woodyatt, L., \& Hedrick, K. (2012). No genuine self-forgiveness without accepting responsibility: Value reaffirmation as a key to maintaining positive self-regard: Genuine self-forgiveness. European Journal of Social Psychology, 42(5), 617-627. https://doi.org/10.1002/ejsp.1873

Werner, K. H., Jazaieri, H., Goldin, P. R., Ziv, M., Heimberg, R. G., \& Gross, J. J. (2012). Self-compassion and social anxiety disorder. Anxiety, Stress, and Coping, 25(5), 543-558. https://doi.org/10.1080/10615806.2011.608842

Wohl, M. J. A., DeShea, L., \& Wahkinney, R. L. (2008). Looking within: Measuring state self-forgiveness and its relationship to psychological well-being. Canadian Journal of Behavioural Science / Revue Canadienne Des Sciences Du Comportement, 40(1), 1-10. https://doi.org/10.1037/0008-400x.40.1.1.1

World Health Organization. (1996). WHOQOL-BREF: Introduction, administration, scoring and generic version of the assessment: field trial version, December 1996. https://apps.who.int/iris/handle/10665/63529

Worthington, E. L. ,. Jr., O'Connor, L. E., Berry, J. W., Sharp, C., Murray, R., \& Yi, E. (2005). Compassion and forgiveness: Implications for psychotherapy. In P. Gilbert (Ed.), Compassion: Conceptualisations, research and use in psychotherapy (pp. 168-192). Routledge.

Worthington, E. L. Jr. (2001). Five steps to forgiveness: The art and science of forgiving. Crown.

Worthington, E. L., \& Scherer, M. (2004). Forgiveness is an emotion-focused coping strategy that can reduce health risks and promote health resilience: Theory, review, and hypotheses. Psychology 83 Health, 19(3), 385-405. https://doi.org/10.1080/0887044042000196674

Zuroff, D. C., Igreja, I., \& Mongrain, M. (1990). Dysfunctional Attitudes, Dependency, and Self-Criticism as predictors of depressive mood states: A 12-month longitudinal study. Cognitive Therapy and Research, 14(3), 315-326. https://doi.org/10.1007/BF01183999 